\title{
SYMMETRIZATION INEQUALITIES FOR COMPOSITION OPERATORS OF CARATHÉODORY TYPE
}

\author{
H. HAJAIEJ AND C. A. STUART
}

\section{Introduction}

Symmetrization (or rearrangement) of a function is used for several purposes in the field of partial differential equations, $[1,2,4,11,12,13]$. One area where it has played a significant role is the study of ground states of scalar field equations of the form

$$
\Delta u+|u|^{\sigma} u+\lambda u=0 \quad \text { for } u \in H^{1}\left(\mathbb{R}^{N}\right) \text { and } \lambda \in \mathbb{R}
$$

where $\sigma>0$. When a ground state exists, symmetrization inequalities show that it is (up to a translation) a Schwarz symmetric function since the energy functional

$$
J(u)=\int_{\mathbb{R}^{N}}\left\{\frac{1}{2}|\nabla u|^{2}-\frac{1}{\sigma+2}|u|^{\sigma+2}\right\} d x
$$

is decreased when $u$ is replaced by its symmetrization $u^{*}$, whereas $\int_{\mathbb{R}^{N}} u^{2} d x$ remains unchanged. Furthermore, symmetrization helps to establish the existence of a ground state. See $[\mathbf{1 0}, \mathbf{1 4}, \mathbf{1 5}]$ for examples of this. For the more general equation

$$
\Delta u(x)+f(|x|, u(x))+\lambda u(x)=0,
$$

the energy functional is

$$
J(u)=\int_{\mathbb{R}^{N}}\left\{\frac{1}{2}|\nabla u|^{2}-F(|x|, u(x))\right\} d x
$$

where $F(r, t)=\int_{0}^{t} f(r, s) d s$ and the extension of the procedure described above rests upon the inequality

$$
\int_{\mathbb{R}^{N}} F(|x|, u(x)) d x \leqslant \int_{\mathbb{R}^{N}} F\left(|x|, u^{*}(x)\right) d x .
$$

In the context of (1.1), it is natural to suppose that $f$, and hence $F$, is a function of Carathéodory type. For a smooth function $F$ (at least $C^{2}$ ) such that $\partial_{1} F \leqslant 0$ and $\partial_{1} \partial_{2} F \leqslant 0$, a version of (1.3) on a bounded open subset $\Omega$ of $\mathbb{R}^{N}$ appears as Lemma 4.6 in [16]. However, we are not aware of any previous results concerning (1.3) in the case where $F$ is not continuous on $(0, \infty) \times[0, \infty)$.

In some circumstances the inequality (1.3) can be deduced from the inequality

$$
\int_{\mathbb{R}^{N}} G(v(x), u(x)) d x \leqslant \int_{\mathbb{R}^{N}} G\left(v^{*}(x), u^{*}(x)\right) d x
$$

by making an appropriate choice of the function $v=v^{*}$. See [3, Corollary 1]. Now the inequality (1.4) has been established in [5] for a class of continuous 
functions $G$ (and it can be obtained for some Borel measurable functions $G,[8]$ ) but we do not believe that this approach can yield (1.3) for functions of Carathéodory type. The purpose of this note is to present a self-contained proof of this useful result in the context of Carathéodory functions $F:(0, \infty) \times[0, \infty) \rightarrow \mathbb{R}$ having the requisite properties. The inequality holds for a broad class $F_{N}$ of nonnegative measurable functions $u$, containing the absolute values of all functions in $\bigcup_{1 \leqslant p<\infty} L^{p}\left(\mathbb{R}^{N}\right)$. Of course, the restriction to non-negative functions can be relaxed by defining the symmetrization of $u$ to be the symmetrization of $|u|$.

In the next section we give a self-contained exposition of the basic notions concerning Schwarz symmetrization that we need. Section 3 deals with simple functions and, as a first application of our approach, we establish the HardyLittlewood inequality for functions in our class $F_{N}$. In $\S 4$, we prove the identity

$$
\int_{\mathbb{R}^{N}} G(u(x)) d x=\int_{\mathbb{R}^{N}} G\left(u^{*}(x)\right) d x
$$

under rather weak assumptions on $G$ and $u$. It is one of the simplest results concerning symmetrization but our assumptions (essentially that $G$ is Borel measurable) are a little weaker than those required in other approaches. For example, (1.5) is stated in [12] for a function $G$ which is the difference of two monotone functions and it is proved for continuous functions $G$ in [5].

Our results concerning (1.3) are given in $\S 5$. The essential ingredients of our approach apply directly to Carathéodory functions having certain monotonicity properties and this leads directly to Proposition 5.1 which establishes (1.3) for all functions $u$ in the class $F_{N}$ which is introduced at the beginning of $\S 2$. We then show that some of the restrictions on $F$ can be relaxed, yielding Theorems 5.3 and 5.4. Results dealing explicitly with functions $F$ of the type appearing in (1.2) are formulated as Corollaries 5.2 and 5.5. Finally, in $\S 6$, we show how our results concerning (1.3) can be used to obtain similar conclusions about functions $u$ defined on subsets of $\mathbb{R}^{N}$ having finite measure. Applications of (1.3) to scalar field equations like (1.1) are presented in [8] which builds on the present approach to deal with the inequality

$$
\int_{\mathbb{R}^{N}} H(|x|, v(x), u(x)) d x \leqslant \int_{\mathbb{R}^{N}} H\left(|x|, v^{*}(x), u^{*}(x)\right) d x
$$

which generalizes simultaneously (1.3) and (1.4).

In an integral where no domain of integration is indicated, it is to be understood that the integration extends over all of $\mathbb{R}^{N}$.

For $r \geqslant 0, B(0, r)=\left\{x \in \mathbb{R}^{N}:|x|<r\right\}$.

\section{Schwarz symmetrization}

From now on all statements about measurability refer to the Lebesgue measure on $\mathbb{R}^{N}$ or on $[0, \infty)$, except in $\S 4$ where we discuss the composition of a Borel measurable function $G$ with a Lebesgue measurable function $u$. If $A$ is a measurable subset of $\mathbb{R}^{N}$, its measure is denoted by $\mu(A)$. There is a constant $V_{N}>0$ such that $\mu(B(0, r))=V_{N} r^{N}$ for all $r>0$.

For a measurable subset $A$ of $\mathbb{R}^{N}$ with $\mu(A)<\infty$,

$$
A^{*}=B(0, r) \quad \text { where } V_{N} r^{N}=\mu(A) .
$$

Note that $A^{*}$ is open even though $A$ may not be. 
The characteristic function of a set $A$ is denoted by $\chi_{A}$.

Let $M_{N}$ denote the set of all extended real-valued functions which are measurable on $\mathbb{R}^{N}$. For $u \in M_{N}$ and $t \in \mathbb{R}$, let

and set

$$
d_{u}(t)=\mu\left(\left\{x \in \mathbb{R}^{N}: u(x)>t\right\}\right)
$$

$$
F_{N}=\left\{u \in M_{N}: 0 \leqslant u<\infty \text { a.e. on } \mathbb{R}^{N} \text { and } d_{u}(t)<\infty \text { for all } t>0\right\} .
$$

For $u \in F_{N}$, the distribution function $d_{u}$ is finite and non-increasing on $(0, \infty)$. (Such functions $u$ are said to vanish weakly at infinity.) Furthermore,

$$
\lim _{t \rightarrow \infty} d_{u}(t)=\mu\left(\bigcap_{t>0}\left\{x \in \mathbb{R}^{N}: u(x)>t\right\}\right)=\mu\left(\left\{x \in \mathbb{R}^{N}: u(x)=\infty\right\}\right)=0
$$

by [9, Theorem 9.E]. Similarly, [9, Theorem 9.D] shows that $d_{u}$ is right-continuous on $(0, \infty)$ and that $d_{u}(0)=\lim _{t \rightarrow 0+} d_{u}(t)$. Note that $d_{u}(0)$ may be infinite.

Definition 2.1. An element $u \in F_{N}$ is said to be Schwarz symmetric if there exists a non-increasing function $h:(0, \infty) \rightarrow[0, \infty)$ such that $u(x)=h(|x|)$ for a.e. $x \in \mathbb{R}^{N}$.

Note that we must have $\lim _{s \rightarrow \infty} h(s)=0$. Furthermore, $\lim _{s \rightarrow 0} h(s)<\infty$ if and only if $u \in F_{N} \cap L^{\infty}\left(\mathbb{R}^{N}\right)$.

We shall show that Schwarz symmetric functions can be modified on a set of measure zero so that the function $h$ is right-continuous.

Lemma 2.1. Let $h, g:(0, \infty) \rightarrow[0, \infty)$ be two functions which are non-increasing and right-continuous on $(0, \infty)$. Suppose that, for all $t>0$,

$$
\mu\left(\left\{x \in \mathbb{R}^{N}: h(|x|)>t\right\}\right)=\mu\left(\left\{x \in \mathbb{R}^{N}: g(|x|)>t\right\}\right) .
$$

Then $h(r)=g(r)$ for all $r \in(0, \infty)$.

Proof. Suppose that there is a number $R>0$ such that $h(R)<g(R)$ and then choose $t \in(h(R), g(R))$. Then $t>0$ and there exists $\delta>0$ such that $g(r)>t$ for all $r \leqslant R+\delta$. This implies that

$$
\left\{x \in \mathbb{R}^{N}: h(|x|)>t\right\} \subset B(0, R)
$$

whereas

$$
B(0, R+\delta) \subset\left\{x \in \mathbb{R}^{N}: g(|x|)>t\right\} .
$$

Thus, for this choice of $t$,

$$
\mu\left(\left\{x \in \mathbb{R}^{N}: h(|x|)>t\right\}\right)<\mu\left(\left\{x \in \mathbb{R}^{N}: g(|x|)>t\right\}\right),
$$

contradicting our assumption. Hence we must have $h(r) \geqslant g(r)$ for all $r>0$. Interchanging the roles of $h$ and $g$ yields the equality of these functions.

This means that every Schwarz symmetric function has a unique normalized form as shown in the next result. 
Proposition 2.2. Let $u \in F_{N}$ be a Schwarz symmetric function. There exists a unique function $\widetilde{u} \in F_{N}$ such that

(a) $\widetilde{u}(x)=H(|x|)$ for all $x \in \mathbb{R}^{N} \backslash\{0\}$ where $H:(0, \infty) \rightarrow[0, \infty)$ is nonincreasing and right-continuous on $(0, \infty)$ and $\widetilde{u}(0)=\lim _{s \rightarrow 0+} H(s)$,

(b) $\widetilde{u}(x)=u(x)$ a.e. on $\mathbb{R}^{N}$.

The function $\widetilde{u}$ is Schwarz symmetric and lower semi-continuous on $\mathbb{R}^{N}$.

Proof. Since $u$ is Schwarz symmetric, there is a non-increasing function $h:(0, \infty) \rightarrow[0, \infty)$ such that $u(x)=h(|x|)$ for a.e. $x \in \mathbb{R}^{N}$. By the monotony of $h$, there exists a countable set $D$ such that $h$ is continuous on $(0, \infty) \backslash D$. The function $H(r)=\lim _{s \rightarrow r+} h(s)$ is right-continuous and non-increasing on $(0, \infty)$. Setting $\widetilde{u}(x)=H(|x|)$ for all $x \in \mathbb{R}^{N} \backslash\{0\}$ and $\widetilde{u}(0)=\lim _{s \rightarrow 0+} H(s)$, we see that (a) holds. Since $H=h$ on $(0, \infty) \backslash D$, it follows that $u(x)=h(|x|)=H(|x|)=\widetilde{u}(x)$ for a.e. $x \in \mathbb{R}^{N}$. This proves (b) which implies that $\widetilde{u} \in F_{N}$. To establish the uniqueness of $\widetilde{u}$, let us consider a function $g$ with the same properties as $H$ such that $u(x)=g(|x|)$ for a.e. $x \in \mathbb{R}^{N}$. Then, for $t>0$,

$$
\begin{aligned}
\mu\left(\left\{x \in \mathbb{R}^{N}: g(|x|)>t\right\}\right) & =\mu\left(\left\{x \in \mathbb{R}^{N}: u(x)>t\right\}\right) \\
& =\mu\left(\left\{x \in \mathbb{R}^{N}: H(|x|)>t\right\}\right)
\end{aligned}
$$

and it follows from Lemma 2.1 that $g \equiv H$, proving the uniqueness of $\widetilde{u}$.

Clearly $\widetilde{u}$ is Schwarz symmetric. For the lower semicontinuity, we need only show that $\left\{x \in \mathbb{R}^{N}: \widetilde{u}(x)>t\right\}$ is open for all $t \in \mathbb{R}$. See [7, p. 218]. For $t>0$,

$$
\left\{x \in \mathbb{R}^{N}: \widetilde{u}(x)>t\right\}=\left\{x \in \mathbb{R}^{N} \backslash\{0\}: H(|x|)>t\right\} \cup\{0\}
$$

if $\lim _{s \rightarrow 0+} H(s)>t$ and

$$
\left\{x \in \mathbb{R}^{N}: \widetilde{u}(x)>t\right\}=\emptyset
$$

if $\lim _{s \rightarrow 0+} H(s) \leqslant t$. Thus for $t>0,\left\{x \in \mathbb{R}^{N}: \widetilde{u}(x)>t\right\}$ is either an open ball or the empty set. Thus $\left\{x \in \mathbb{R}^{N}: \widetilde{u}(x)>0\right\}=\bigcup_{t>0}\left\{x \in \mathbb{R}^{N}: \widetilde{u}(x)>t\right\}$ is also open. For $t<0,\left\{x \in \mathbb{R}^{N}: \widetilde{u}(x)>t\right\}=\mathbb{R}^{N}$. Hence $\widetilde{u}$ is lower semi-continuous on $\mathbb{R}^{N}$.

Schwarz symmetrization associates with every element $u \in F_{N}$ a Schwarz symmetric function $u^{*}$ having the same distribution function as $u$. With the normalization shown in Proposition 2.2, the element $u^{*}$ is uniquely determined by $u$. There are several ways of showing that this kind of symmetrization is possible. We use one of them and then we establish the properties of symmetrization that are required for the proof of our main results.

Given $u \in F_{N}$, we can define a function $h_{u}:(0, \infty) \rightarrow[0, \infty)$ by setting

$$
h_{u}(s)=\inf \left\{t \geqslant 0: d_{u}(t) \leqslant V_{N} s^{N}\right\} \quad \text { for } s>0 .
$$

The set $\left\{t \geqslant 0: d_{u}(t) \leqslant V_{N} s^{N}\right\}$ is not empty since $d_{u}(t) \rightarrow 0$ as $t \rightarrow \infty$, and by the monotonicity and right-continuity of $d_{u},\left\{t \geqslant 0: d_{u}(t) \leqslant V_{N} s^{N}\right\}=\left[h_{u}(s), \infty\right)$. Clearly $h_{u}$ is non-increasing on $(0, \infty)$. Setting $h_{u}(0)=\lim _{s \rightarrow 0+} h_{u}(s)$, we observe that $h_{u}(0)<\infty$ if and only if $u \in F_{N} \cap L^{\infty}\left(\mathbb{R}^{N}\right)$. 
Lemma 2.3. For every $u \in F_{N}$,

(i) $h_{u}$ is right-continuous on $(0, \infty)$ and

(ii) for all $s, t \geqslant 0, h_{u}(s)>t$ if and only if $d_{u}(t)>V_{N} s^{N}$.

Proof. (i) Consider $s>0$ and a decreasing sequence $\left\{s_{k}\right\} \subset(s, \infty)$ such that $s_{k} \rightarrow s$. Then, $\left\{h_{u}\left(s_{k}\right)\right\}$ is a non-decreasing sequence with $h_{u}\left(s_{k}\right) \leqslant h_{u}(s)$ for all $k \in \mathbb{N}$. Let $L=\lim h_{u}\left(s_{k}\right)$. Clearly $L \leqslant h_{u}(s)$. But $L \geqslant h_{u}\left(s_{k}\right)$ and so $d_{u}(L) \leqslant V_{N} s_{k}^{N}$ for all $k \in \mathbb{N}$. Thus $d_{u}(L) \leqslant V_{N} s^{N}$ showing that $h_{u}(s) \leqslant L$.

(ii) Suppose that $h_{u}(s)>t$. If $s>0$, this implies that $d_{u}(t)>V_{N} s^{N}$ by the definition of $h_{u}$. If $s=0$, there exists $\tau>0$ such that $h_{u}(\tau)>t$ since $h_{u}(0)$ was defined to be $\lim _{s \rightarrow 0+} h_{u}(s)$. Thus $d_{u}(t)>V_{N} \tau^{N}>0$ when $s=0$.

Conversely, suppose that $d_{u}(t)>V_{N} s^{N}$. If $s>0$, the right-continuity of $d_{u}$ implies that $t<h_{u}(s)$. For $s=0$, we have $d_{u}(t)>0$ and hence there exists $\tau>0$ such that $d_{u}(t)>V_{N} \tau^{N}$. Thus $h_{u}(\tau)>t$ and consequently, $h_{u}(0)>t$ by the monotonicity of $h_{u}$.

Definition 2.2. The Schwarz symmetrization of an element $u \in F_{N}$ is the function $u^{*}$ defined by

$$
u^{*}(x)=h_{u}(|x|) .
$$

If $v=u$ a.e. on $\mathbb{R}^{N}$, then $v^{*}=u^{*}$. The next result shows that $u^{*}$ is the unique normalized (in the sense of Proposition 2.2) Schwarz symmetric function having the same distribution function as $u$.

Proposition 2.4. Consider $u \in F_{N}$.

(i) For all $t>0, \quad\left\{x \in \mathbb{R}^{N}: u^{*}(x)>t\right\}=\left\{x \in \mathbb{R}^{N}: u(x)>t\right\}^{*}=B(0, r)$ where $V_{N} r^{N}=d_{u}(t)$. Also, $d_{u^{*}}(t)=d_{u}(t)$ for all $t \geqslant 0$.

(ii) The function $u^{*} \in F_{N}, u^{*}$ is Schwarz symmetric and $u^{*}$ is lower semicontinuous on $\mathbb{R}^{N}$.

(iii) If $u$ is Schwarz symmetric, $u^{*}=u$ a.e. on $\mathbb{R}^{N}$.

(iv) If $v \in F_{N}$ with $u \leqslant v$ a.e. on $\mathbb{R}^{N}$, then $u^{*} \leqslant v^{*}$ on $\mathbb{R}^{N}$.

(v) If $\left\{u_{k}\right\} \subset F_{N}$ with $u_{k} \leqslant u_{k+1}$ and $\lim _{k \rightarrow \infty} u_{k}=u$ on $\mathbb{R}^{N}$, then

$$
\lim _{k \rightarrow \infty} u_{k}^{*}=u^{*} \quad \text { on } \mathbb{R}^{N} \text {. }
$$

Proof. (i) For $t>0$,

$$
\left\{x \in \mathbb{R}^{N}: u^{*}(x)>t\right\}=\left\{x \in \mathbb{R}^{N}: h_{u}(|x|)>t\right\} .
$$

Now, $h_{u}(|x|)>t$ if and only if $d_{u}(t)>V_{N}|x|^{N}$ by Lemma 2.3(ii), and so

$$
\left\{x \in \mathbb{R}^{N}: u^{*}(x)>t\right\}=\left\{x \in \mathbb{R}^{N}: d_{u}(t)>V_{N}|x|^{N}\right\}=B(0, r)
$$

and $V_{N} r^{N}=d_{u}(t)$. But, by definition, $\left\{x \in \mathbb{R}^{N}: u(x)>t\right\}^{*}=B(0, r)$ where $V_{N} r^{N}=\mu\left(\left\{x \in \mathbb{R}^{N}: u(x)>t\right\}\right)$. Since $d_{u}(t)=\mu\left(\left\{x \in \mathbb{R}^{N}: u(x)>t\right\}\right)$, it follows that $\left\{x \in \mathbb{R}^{N}: u^{*}(x)>t\right\}=\left\{x \in \mathbb{R}^{N}: u(x)>t\right\}^{*}$ for $t>0$. Since $\mu\left(\left\{x \in \mathbb{R}^{N}: u(x)>t\right\}^{*}\right)=$ $\mu\left(\left\{x \in \mathbb{R}^{N}: u(x)>t\right\}\right)$, this proves that

$$
d_{u^{*}}(t)=\mu\left(\left\{x \in \mathbb{R}^{N}: u^{*}(x)>t\right\}\right)=\mu\left(\left\{x \in \mathbb{R}^{N}: u(x)>t\right\}\right)=d_{u}(t)
$$


for all $t>0$. Finally, $d_{u^{*}}(0)=\lim _{t \rightarrow 0+} d_{u^{*}}(t)=\lim _{t \rightarrow 0+} d_{u}(t)=d_{u}(0)$, as noted just before Definition 2.1.

(ii) The lower semicontinuity of $u^{*}$ follows from the same arguments as in Proposition 2.2. These properties also show that $u^{*}$ is measurable on $\mathbb{R}^{N}$. Since $d_{u^{*}}(t)=d_{u}(t)<\infty$ for all $t>0$ and $0 \leqslant u^{*}(x)<\infty$ for $x \neq 0$, it follows that $u^{*} \in F_{N}$. Clearly $u^{*}$ is Schwarz symmetric.

(iii) Suppose that $u$ is Schwarz symmetric. Then, by Proposition 2.2, there exists a non-increasing right-continuous function $H:(0, \infty) \rightarrow[0, \infty)$ such that $u(x)=H(|x|)$ for a.e. $x \in \mathbb{R}^{N}$. On the other hand, $h_{u}:(0, \infty) \rightarrow[0, \infty)$ is also a non-increasing, right-continuous function and, for any $t>0$,

$$
\begin{aligned}
\mu\left(\left\{x \in \mathbb{R}^{N}: h_{u}(|x|)>t\right\}\right) & =\mu\left(\left\{x \in \mathbb{R}^{N}: u^{*}(x)>t\right\}\right) \\
& =d_{u^{*}}(t)=d_{u}(t) \\
& =\mu\left(\left\{x \in \mathbb{R}^{N}: u(x)>t\right\}\right) \\
& =\mu\left(\left\{x \in \mathbb{R}^{N}: H(|x|)>t\right\}\right)
\end{aligned}
$$

by part (i). It follows from Lemma 2.1 that $h_{u} \equiv H$ and hence that $u^{*}=u$ a.e. on $\mathbb{R}^{N}$.

(iv) For any $t \geqslant 0,\left\{x \in \mathbb{R}^{N}: u(x)>t\right\} \subset\left\{x \in \mathbb{R}^{N}: v(x)>t\right\} \cup A$ where $\mu(A)=0$ and so $d_{u}(t) \leqslant d_{v}(t)$. Then, for any $s>0$,

$$
\left\{t \geqslant 0: d_{v}(t) \leqslant V_{N} s^{N}\right\} \subset\left\{t \geqslant 0: d_{u}(t) \leqslant V_{N} s^{N}\right\}
$$

and hence $h_{u}(s) \leqslant h_{v}(s)$. Thus $h_{u}(0) \leqslant h_{v}(0)$ too and we have $u^{*} \leqslant v^{*}$ on $\mathbb{R}^{N}$.

(v) By part (iv), $u_{k}^{*} \leqslant u_{k+1}^{*} \leqslant u^{*}$. Fix $t \geqslant 0$ and set $A_{k}=\left\{x \in \mathbb{R}^{N}: u_{k}(x)>t\right\}$. Then $A_{k} \subset A_{k+1}$ and $\bigcup_{k \in \mathbb{N}} A_{k}=\left\{x \in \mathbb{R}^{N}: u(x)>t\right\}$. By [9, Theorem 9.D],

$$
d_{u}(t)=\mu\left(\left\{x \in \mathbb{R}^{N}: u(x)>t\right\}\right)=\lim _{k \rightarrow \infty} \mu\left(A_{k}\right)=\lim _{k \rightarrow \infty} d_{u_{k}}(t) .
$$

Now the proof of part (iv) shows that $h_{u_{k}} \leqslant h_{u_{k}+1} \leqslant h_{u}$. Thus

$$
\lim _{k \rightarrow \infty} h_{u_{k}}(s) \leqslant h_{u}(s)
$$

for all $s \geqslant 0$. Suppose that $h_{u}(s)>0$ and consider $T \in\left[0, h_{u}(s)\right)$. By Lemma 2.3(ii), $d_{u}(T)>V_{N} s^{N}$. Hence there exists $k \in \mathbb{N}$ such that $d_{u_{k}}(T)>V_{N} s^{N}$ and so Lemma 2.3(ii) now yields $h_{u_{k}}(s)>T$. Thus $\lim _{k \rightarrow \infty} h_{u_{k}}(s)>T$ and we have shown that $\lim _{k \rightarrow \infty} h_{u_{k}}(s)=h_{u}(s)$ for all $s \geqslant 0$. It follows immediately that

$$
\lim _{k \rightarrow \infty} u_{k}^{*}(x)=u^{*}(x) \text { for all } x \in \mathbb{R}^{N} \text {. }
$$

\section{Simple functions}

The symmetrization of simple functions can be done in a very direct manner. Recall that a simple function is a measurable function whose range consists of a finite number of points in $\mathbb{R}$. Let

$$
E_{N}=\left\{u \in F_{N}: u \text { is a simple function }\right\} .
$$

That is to say, $E_{N}$ is the set of all functions which can be written as

$$
u=\sum_{i=0}^{k} a_{i} \chi_{A_{i}} \quad \text { for some } k \in \mathbb{N}
$$


where $a_{i} \in(0, \infty)$ with $a_{i}>a_{i+1}, \quad A_{i}$ is a measurable subset of $\mathbb{R}^{N}$ with $\mu\left(A_{i}\right)<\infty$ and $A_{i} \cap A_{j}=\emptyset$ for $i \neq j$.

We now show that the Schwarz symmetrization of an element in $E_{N}$ is also a simple function. Let $u$ be the function (3.1) and set

$$
S_{i}=\bigcup_{j=0}^{i} A_{j} \quad \text { and } \quad S_{i}^{*}=B\left(0, r_{i}\right) \text { for } 0 \leqslant i \leqslant k
$$

Then $0 \leqslant r_{0} \leqslant \ldots \leqslant r_{k}<\infty$ and it is easy to check that

$$
d_{u}=\sum_{i=0}^{k}\left[\sum_{j=0}^{i} \mu\left(A_{j}\right)\right] \chi_{\left[a_{i+1}, a_{i}\right)} \quad \text { with } a_{k+1}=0
$$

and then that

$$
h_{u}=\sum_{i=0}^{k} a_{i} \chi_{\left[r_{i-1}, r_{i}\right)} \quad \text { where } r_{-1}=0 .
$$

We note that $r_{i-1}<r_{i}$ if and only if $\mu\left(A_{i}\right)>0$. It follows that

$$
u^{*}=\sum_{i=0}^{k} a_{i} \chi_{C_{i}}
$$

where $C_{0}=B\left(0, r_{0}\right)$ and $C_{i}=B\left(0, r_{i}\right) \backslash \overline{B\left(0, r_{i-1}\right)}$ for $i=1, \ldots, k$. Thus $C_{i} \neq \emptyset$ if and only if $\mu\left(A_{i}\right)>0$, and so the range of $u^{*}$ is $\left\{a_{i}: \mu\left(A_{i}\right)>0\right\} \cup\{0\}$.

The symmetrization of a simple function can be expressed in an alternative way which is very convenient for some purposes. It is a consequence of the following simple lemma which will be used several times in $\S 4$.

Lemma 3.1. Let $\left\{p_{i}: i=0,1, \ldots, k\right\} \subset \mathbb{R}$ and $\left\{q_{i}: i=0,1, \ldots, k\right\} \subset \mathbb{R}$. Then

$$
\sum_{i=0}^{k} p_{i} q_{i}=\sum_{i=0}^{k} P_{i} Q_{i}
$$

where $P_{i}=p_{i}-p_{i+1}$ for $i=0,1, \ldots, k-1, P_{k}=p_{k}$ and $Q_{i}=\sum_{j=0}^{i} q_{j}$.

Proof. We have

$$
\sum_{i=0}^{k} P_{i} Q_{i}=\sum_{i=0}^{k} P_{i} \sum_{j=0}^{i} q_{j}=\sum_{j=0}^{k} q_{j} \sum_{i=j}^{k} P_{i}=\sum_{j=0}^{k} q_{j} p_{j} .
$$

Consider now a simple function expressed as (3.1). Using (3.4), we have

$$
u=\sum_{i=0}^{k} a_{i} \chi_{A_{i}}=\sum_{i=0}^{k} \alpha_{i} \sum_{j=0}^{i} \chi_{A_{i}}=\sum_{i=0}^{k} \alpha_{i} \chi_{S_{i}}
$$

where $\alpha_{i}=a_{i}-a_{i+1}$ for $i=0,1, \ldots, k$ (recall that $a_{k+1}=0$ ) and $S_{i}$ is defined by (3.2). Similarly, using (3.3), we have

$$
u^{*}=\sum_{i=0}^{k} a_{i} \chi_{C_{i}}=\sum_{i=0}^{k} \alpha_{i} \sum_{j=0}^{i} \chi_{C_{i}}=\sum_{i=0}^{k} \alpha_{i} \chi_{B_{i}}
$$


where $B_{i}=B\left(0, r_{i}\right)=\bigcup_{j=0}^{i} C_{j}$. Observing that $S_{i}^{*}=B_{i}$ by (3.2), we see from (3.1) and (3.3) that

$$
\left(\chi_{S_{i}}\right)^{*}=\chi_{B_{i}} \text { for } i=0,1, \ldots, k .
$$

Hence the expressions (3.5) and (3.6) show that

$$
\left(\sum_{i=0}^{k} \alpha_{i} \chi_{S_{i}}\right)^{*}=\sum_{i=0}^{k} \alpha_{i}\left(\chi_{S_{i}}\right)^{*}=\sum_{i=0}^{k} \alpha_{i} \chi_{S_{i}^{*}} .
$$

These attractive formulae for simple functions become more consequential when we recall that every positive measurable function is the limit of an increasing sequence of simple functions.

Proposition 3.2. Let $u \in F_{N}$. There is a sequence $\left\{u_{k}\right\} \subset E_{N}$ such that

$$
u_{k} \leqslant u_{k+1} \quad \text { and } \quad \lim _{k \rightarrow \infty} u_{k}=u \quad \text { on } \mathbb{R}^{N} \text {. }
$$

This result is a minor adaptation of [9, Theorem 20.B], for example.

These considerations lead to a short proof (see also [10]) of the following famous result which we need later.

Proposition 3.3 (Hardy-Littlewood inequality). For all $u, v \in F_{N}$,

$$
\int u(x) v(x) d x \leqslant \int u^{*}(x) v^{*}(x) d x .
$$

Proof. Suppose first that $u, v \in E_{N}$ and that, in the notation (3.5),

$$
u=\sum_{i=0}^{k} \alpha_{i} \chi_{S_{i}} \quad \text { and } \quad v=\sum_{j=0}^{m} \beta_{j} \chi_{T_{j}} .
$$

Then, by (3.6),

$$
u^{*}=\sum_{i=0}^{k} \alpha_{i} \chi_{B_{i}} \quad \text { and } \quad v^{*}=\sum_{j=0}^{m} \beta_{j} \chi_{D_{j}}
$$

where $S_{i}^{*}=B_{i}$ and $T_{j}^{*}=D_{j}$. Thus,

$$
\int u v d x=\sum_{i=0}^{k} \sum_{j=0}^{m} \alpha_{i} \beta_{j} \int \chi_{S_{i}} \chi_{T_{j}} d x
$$

and

$$
\int u^{*} v^{*} d x=\sum_{i=0}^{k} \sum_{j=0}^{m} \alpha_{i} \beta_{j} \int \chi_{B_{i}} \chi_{D_{j}} d x .
$$

But,

$$
\int \chi_{S_{i}} \chi_{T_{j}} d x=\mu\left(S_{i} \cap T_{j}\right) \leqslant \min \left\{\mu\left(S_{i}\right), \mu\left(T_{j}\right)\right\}
$$

whereas

$$
\int \chi_{B_{i}} \chi_{D_{j}} d x=\min \left\{\mu\left(B_{i}\right), \mu\left(D_{j}\right)\right\}
$$


since $B_{i}$ and $D_{j}$ are open balls centered at the origin. Recalling that $\mu\left(B_{i}\right)=\mu\left(S_{i}\right)$ and $\mu\left(D_{j}\right)=\mu\left(T_{j}\right)$, we see that

$$
\int u v d x \leqslant \int u^{*} v^{*} d x \text { for all } u, v \in E_{N}
$$

since $\alpha_{i} \beta_{j}>0$ for all $i=0,1 \ldots, k$ and $j=0,1, \ldots, m$.

Using Proposition 3.2 and Proposition 2.4(v), we see that the Monotone Convergence Theorem (see [9, Theorem 27.B], for example) yields the same inequality for all $u, v \in F_{N}$.

\section{Composition with a Borel function}

In this section we consider $G \circ u$ and $G \circ u^{*}$ where $u \in F_{N}$ and $G$ is a realvalued Borel measurable function on $[0, \infty)$. In this case, $G \circ u$ and $G \circ u^{*}$ are both (Lebesgue) measurable on $\mathbb{R}^{N}$. Indeed, there is a set $A \subset \mathbb{R}^{N}$ (depending on $u$ ) such that $\mu(A)=0$ and $u(x) \in[0, \infty)$ for all $x \in \mathbb{R}^{N} \backslash A$. Now, for any $t \in \mathbb{R}$,

$$
\left\{x \in \mathbb{R}^{N} \backslash A: G(u(x))>t\right\}=\left\{x \in \mathbb{R}^{N}: u(x) \in G^{-1}((t, \infty))\right\}
$$

and $G^{-1}((t, \infty))$ is a Borel subset of $\mathbb{R}$. This proves that $G \circ u$ is measurable on $\mathbb{R}^{N} \backslash A$ and hence, by an arbitrary extension, it can be considered to be measurable on all $\mathbb{R}^{N}$. Clearly the same is true for $G \circ u^{*}$. It is well known that the Borel measurability of $G$ cannot be replaced by Lebesgue measurability in these statements.

In this context we shall establish the identity

$$
\int G(u(x)) d x=\int G\left(u^{*}(x)\right) d x
$$

under rather weak additional restrictions on $G$ or $u$, including cases where the integrals are infinite. We begin with the case where $G$ is positive and then extend the conclusion to the general case in a standard way. The following property of Schwarz symmetrization is crucial for our approach.

Lemma 4.1. Let $u \in F_{N}$ and let $A$ be a Borel subset of $(0, \infty)$. Then

$$
\mu\left(\left\{x \in \mathbb{R}^{N}: u(x) \in A\right\}\right)=\mu\left(\left\{x \in \mathbb{R}^{N}: u^{*}(x) \in A\right\}\right) .
$$

Proof. By Proposition 2.4(i), $d_{u}=d_{u^{*}}$ and so

$$
\mu\left(\left\{x \in \mathbb{R}^{N}: u(x)>t\right\}\right)=\mu\left(\left\{x \in \mathbb{R}^{N}: u^{*}(x)>t\right\}\right)
$$

for all $t \in \mathbb{R}$ since $u$ and $u^{*}$ are non-negative.

Let $\Phi$ denote the set of all intervals of the form

$$
(a, b],(a, \infty) \text { and } \emptyset
$$

where $0 \leqslant a<b<\infty$. In the same way as on [7, p.33], the collection of all finite disjoint unions of elements of $\Phi$ is an algebra of sets, which we denote by $\Psi$, and furthermore the $\sigma$-algebra generated by $\Psi$ is the collection of all Borel subsets of $(0, \infty)$.

We now define two functions $m$ and $M$ on the $\sigma$-algebra of Borel sets of $(0, \infty)$ by setting

$$
m(A)=\mu\left(\left\{x \in \mathbb{R}^{N}: u(x) \in A\right\}\right) \quad \text { and } \quad M(A)=\mu\left(\left\{x \in \mathbb{R}^{N}: u^{*}(x) \in A\right\}\right)
$$


for any Borel subset $A$ of $(0, \infty)$. Since $\mu$ is a measure on $\mathbb{R}^{N}$, it is easy to check that $m$ and $M$ are both Borel measures on $(0, \infty)$. Consequently, they are both premeasures on $\Psi$. That is to say, $m(\emptyset)=0$ and, if $\left\{A_{j}: j \in \mathbb{N}\right\}$ is a sequence of disjoint sets in $\Psi$ such that $\bigcup_{j \in \mathbb{N}} A_{j} \in \Psi$, then $m\left(\bigcup_{j \in \mathbb{N}} A_{j}\right)=\sum_{j \in \mathbb{N}} m\left(A_{j}\right)$; and $M$ has the same properties. Furthermore, since $\mu\left(\left\{x \in \mathbb{R}^{N}: u(x)>1 / n\right\}\right)<\infty$ for all $n \in \mathbb{N}, m$ is $\sigma$-finite on $\Psi$. By [7, Theorem 1.14], there exists a unique Borel measure $\rho$ on $(0, \infty)$ such that $\rho(A)=m(A)$ for all $A \in \Psi$.

Using (4.2), one can easily check that $m(A)=M(A)$ for all $A \in \Phi$ and hence for all $A \in \Psi$. Thus, since we have already noted that $m$ and $M$ are both Borel measures on $(0, \infty)$, it follows that $\rho=m=M$.

REMark. The lemma cannot be extended to all Borel subsets of $[0, \infty)$ as the following example shows. Let $w: \mathbb{R} \rightarrow[0, \infty)$ be defined by

$$
w(x)=e^{-x} \chi_{[0, \infty)}(x) \text { for } x \in \mathbb{R} .
$$

Clearly $w \in F_{1}$ and $\mu(\{x \in \mathbb{R}: w(x)=0\})=\infty$. However, $w^{*}(x)=e^{-2|x|}$ for all $x \in \mathbb{R}$ and so $\mu\left(\left\{x \in \mathbb{R}: w^{*}(x)=0\right\}\right)=0$. This function $w$ also shows that the equality (4.1) cannot hold for all Borel functions $G$. Indeed, setting $G=\chi_{\{0\}}$, we see that $G:[0, \infty) \rightarrow[0, \infty)$ is a Borel function, but

$$
\int G(w(x)) d x=\infty \text { whereas } \int G\left(w^{*}(x)\right) d x=0 .
$$

With this example in mind we establish a preliminary result.

Lemma 4.2. Let $G:[0, \infty) \rightarrow[0, \infty)$ be a Borel measurable function. Then

$$
\int_{\{x: u(x)>0\}} G(u(x)) d x=\int_{\left\{x: u^{*}(x)>0\right\}} G\left(u^{*}(x)\right) d x
$$

for all $u \in F_{N}$.

Proof. Let $g=\left.G\right|_{(0, \infty)}$. By [9, Theorem 20B], there is an increasing sequence $\left\{g_{n}\right\}$ of non-negative Borel measurable simple functions on $(0, \infty)$ such that $g_{n}(s) \rightarrow g(s)$ for all $s>0$. Let

$$
g_{n}=\sum_{i=1}^{k} a_{i} \chi_{A_{i}}
$$

where $a_{i} \in(0, \infty)$ with $a_{i} \neq a_{j}$ when $i \neq j$ and $A_{i}$ is a Borel measurable subset of $(0, \infty)$ with $A_{i} \cap A_{j}=\emptyset$ when $i \neq j$. Then

$$
\int_{\{x: u(x)>0\}} G(u(x)) d x=\int_{\{x: u(x)>0\}} g(u(x)) d x=\lim _{n \rightarrow \infty} \int_{\{x: u(x)>0\}} g_{n}(u(x)) d x
$$

by the Monotone Convergence Theorem, [9, Theorem 27B] for example. Similarly,

But,

$$
\int_{\left\{x: u^{*}(x)>0\right\}} G\left(u^{*}(x)\right) d x=\lim _{n \rightarrow \infty} \int_{\left\{x: u^{*}(x)>0\right\}} g_{n}\left(u^{*}(x)\right) d x .
$$

$$
\int_{\{x: u(x)>0\}} g_{n}(u(x)) d x=\sum_{i=1}^{k} a_{i} \mu\left(\left\{x \in \mathbb{R}^{N}: u(x) \in A_{i}\right\}\right)
$$


and

$$
\int_{\left\{x: u^{*}(x)>0\right\}} g_{n}\left(u^{*}(x)\right) d x=\sum_{i=1}^{k} a_{i} \mu\left(\left\{x \in \mathbb{R}^{N}: u^{*}(x) \in A_{i}\right\}\right),
$$

so the result now follows from Lemma 4.1.

Proposition 4.3. Let $G:[0, \infty) \rightarrow[0, \infty)$ be a Borel measurable function.

(i) We have

$$
\int G(u(x)) d x \geqslant \int G\left(u^{*}(x)\right) d x \text { for all } u \in F_{N} .
$$

(ii) If $G(0)=0$ then

$$
\int G(u(x)) d x=\int G\left(u^{*}(x)\right) d x \text { for all } u \in F_{N} .
$$

Proof. First we note that

$$
\int G(u(x)) d x=\int_{\{x: u(x)>0\}} G(u(x)) d x+G(0) \mu\left(\left\{x \in \mathbb{R}^{N}: u(x)=0\right\}\right)
$$

where it is understood that $G(0) \mu\left(\left\{x \in \mathbb{R}^{N}: u(x)=0\right\}\right)=0$ when $G(0)=0$, even if $\mu\left(\left\{x \in \mathbb{R}^{N}: u(x)=0\right\}\right)=\infty$. Similarly

$$
\int G\left(u^{*}(x)\right) d x=\int_{\left\{x: u^{*}(x)>0\right\}} G\left(u^{*}(x)\right) d x+G(0) \mu\left(\left\{x \in \mathbb{R}^{N}: u^{*}(x)=0\right\}\right) .
$$

Thus part (ii) follows immediately from the preceding lemma. Suppose now that $G(0)>0$. If $\mu\left(\left\{x \in \mathbb{R}^{N}: u(x)=0\right\}\right)=\infty$, then $\int G(u(x)) d x=\infty$ and the inequality (i) certainly holds. If $\mu\left(\left\{x \in \mathbb{R}^{N}: u(x)=0\right\}\right)<\infty$, we claim that $\mu\left(\left\{x \in \mathbb{R}^{N}: u^{*}(x)=0\right\}\right)=0$ and so the inequality (i) follows from the lemma. To justify our claim, we observe that if $z \in\left\{x \in \mathbb{R}^{N}: u^{*}(x)=0\right\}$, then $u^{*}(x)=0$ for all $x \in \mathbb{R}^{N} \backslash B(0,|z|)$. Hence,

and

$$
\mu\left(\left\{x \in \mathbb{R}^{N}: u^{*}(x)>0\right\}\right) \leqslant \mu(B(0,|z|))<\infty
$$

$$
\mu\left(\left\{x \in \mathbb{R}^{N}: u^{*}(x)=0\right\}\right)=\infty .
$$

But, by Proposition 2.4(i), we know that

$$
\mu\left(\left\{x \in \mathbb{R}^{N}: u^{*}(x)>0\right\}\right)=\mu\left(\left\{x \in \mathbb{R}^{N}: u(x)>0\right\}\right)
$$

and so

$$
\mu\left(\left\{x \in \mathbb{R}^{N}: u(x)=0\right\}\right)=\infty
$$

since

$$
\mathbb{R}^{N}=\left\{x \in \mathbb{R}^{N}: u(x)>0\right\} \cup\left\{x \in \mathbb{R}^{N}: u(x)=0\right\} .
$$

Thus if $\mu\left(\left\{x \in \mathbb{R}^{N}: u(x)=0\right\}\right)<\infty$, we must have $\left\{x \in \mathbb{R}^{N}: u^{*}(x)=0\right\}=\emptyset$, establishing the claim. 
REMARK. The proof of part (i) shows that in fact

$$
\int G(u(x)) d x>\int G\left(u^{*}(x)\right) d x
$$

whenever $G(0)>0$ and $u \in F_{N}$ is such that

$$
\int G(u(x)) d x<\infty \quad \text { and } \quad 0<\mu\left(\left\{x \in \mathbb{R}^{N}: u(x)=0\right\}\right) .
$$

We now extend the second part of this result to non-positive functions $G$ in the usual way. Let

$$
G_{+}(s)=\max \{G(s), 0\} \quad \text { and } \quad G_{-}(s)=\max \{-G(s), 0\} .
$$

If $G:[0, \infty) \rightarrow \mathbb{R}$ is a Borel measurable function, then $G_{+}$and $G_{-}$are both nonnegative Borel measurable functions on $[0, \infty)$ and $G=G_{+}-G_{-}$.

THEOREM 4.4. Let $G:[0, \infty) \rightarrow \mathbb{R}$ be a Borel measurable function with $G(0)=0$. Then the equality (4.1) holds for all $u \in F_{N}$ such that at least one of the integrals

$$
\int G_{+}(u(x)) d x \text { or } \int G_{-}(u(x)) d x \text { is finite. }
$$

Proof. For all $u \in F_{N}$, the previous result shows that

$$
\int G_{ \pm}(u(x)) d x=\int G_{ \pm}\left(u^{*}(x)\right) d x .
$$

If $\int G_{+}(u(x)) d x<\infty$, it follows that $\int G_{+}\left(u^{*}(x)\right) d x<\infty$ and so we can write

$$
\begin{aligned}
\int G(u(x)) d x & =\int G_{+}(u(x)) d x-\int G_{-}(u(x)) d x \\
& =\int G_{+}\left(u^{*}(x)\right) d x-\int G_{-}\left(u^{*}(x)\right) d x \\
& =\int G\left(u^{*}(x)\right) d x .
\end{aligned}
$$

The case where $\int G_{-}(u(x)) d x<\infty$ is similar.

\section{Composition with a Carathéodory function}

We begin by recalling the notion of Carathéodory function in the context of our discussion.

Definition 5.1. A function $F:(0, \infty) \times[0, \infty) \rightarrow \mathbb{R}$ is called a Carathéodory function when

(1) $F(\cdot, s):(0, \infty) \rightarrow \mathbb{R}$ is measurable on $(0, \infty)$ for all $s \geqslant 0$, and

(2) $F(r, \cdot):[0, \infty) \rightarrow \mathbb{R}$ is continuous on $[0, \infty)$ for all $r \in(0, \infty) \backslash \Gamma$ where $\Gamma$ is a subset of $(0, \infty)$ having one-dimensional measure zero.

An important property of such a function is that the composition $x \mapsto F(|x|, u(x))$ is measurable on $\mathbb{R}^{N}$ for every function $u \in F_{N^{N}}$. (See [6, Theorem 2.1], for example.) In fact, for $u \in F_{N}$ there is a set $A \subset \mathbb{R}^{N}$ such that 
$\mu(A)=0$ and $0 \leqslant u(x)<\infty$ for all $x \in \mathbb{R}^{N} \backslash A$. Thus $F(|x|, u(x))$ is defined and measurable on $\mathbb{R}^{N} \backslash[A \cup\{0\}]$ and consequently, it is measurable on $\mathbb{R}^{N}$.

Proposition 5.1. Let $F:(0, \infty) \times[0, \infty) \rightarrow \mathbb{R}$ be a Carathéodory function such that

(i) $F(r, 0)=0$ for all $r \in(0, \infty) \backslash \Gamma$,

(ii) for all $r, R \in(0, \infty) \backslash \Gamma$ with $R \geqslant r$ and all $a, b \in[0, \infty)$ with $b \geqslant a$,

$$
F(R, b)-F(R, a)-F(r, b)+F(r, a) \leqslant 0,
$$

(iii) $F(r, \cdot):[0, \infty) \rightarrow \mathbb{R}$ is non-decreasing on $[0, \infty)$ for all $r \in(0, \infty) \backslash \Gamma$.

Then the inequalities

$$
0 \leqslant \int F(|x|, u(x)) d x \leqslant \int F\left(|x|, u^{*}(x)\right) d x
$$

hold for all $u \in F_{N}$.

REMARK 1. Note that the properties (i) and (iii) imply that

$$
F(r, s) \geqslant 0 \quad \text { for all } r \in(0, \infty) \backslash \Gamma \text { and all } s \geqslant 0
$$

and that (i) and (ii) imply that

$$
F(r, s) \geqslant F(R, s) \text { for all } r, R \in(0, \infty) \backslash \Gamma \text { with } R \geqslant r \text { and all } s \geqslant 0 \text {. }
$$

Thus $F(r, s)$ is non-negative, non-increasing in $r$ and non-decreasing in $s$.

REMARK 2. Under the hypotheses of the proposition, the function $F\left(|\cdot|, u^{*}(\cdot)\right)$ is easily seen to be Schwarz symmetric. However, except in trivial cases, it is not the symmetrization of the function $F(|\cdot|, u(\cdot))$. The subsequent theorems in this section deal with weaker assumptions on $F$ under which it is no longer true that $F\left(|\cdot|, u^{*}(\cdot)\right)$ is Schwarz symmetric.

Proof. We begin by establishing the inequality (1.3) for elements of $E_{N}$. Let $u$ be a function of the form (3.1). Then

$$
\int F(|x|, u(x)) d x=\int \sum_{i=0}^{k} F\left(|x|, a_{i}\right) \chi_{A_{i}}(x) d x
$$

since $F(|x|, 0)=0$ and $A_{i} \cap A_{j}=\emptyset$ for $i \neq j$. Now by (3.4),

$$
\sum_{i=0}^{k} F\left(|x|, a_{i}\right) \chi_{A_{i}}(x)=\sum_{i=0}^{k} g_{i}(x) \chi_{S_{i}}(x)
$$

where $S_{i}=\bigcup_{j=0}^{i} A_{j}$ and $g_{i}(x)=h_{i}(|x|)$ with

$$
h_{i}(r)=F\left(r, a_{i}\right)-F\left(r, a_{i+1}\right) \quad \text { for } i=0,1, \ldots, k .
$$

Recall that $a_{k+1}=0$. Using the properties (ii) and (iii) of $F$, we see that, for $i=0,1, \ldots, k$, the functions $h_{i}$ have the following properties:

(i) $h_{i}(r) \geqslant 0$ for $r \in(0, \infty) \backslash \Gamma$ and

(ii) $h_{i}(r) \geqslant h_{i}(R)$ for all $r, R \in(0, \infty) \backslash \Gamma$ with $R \geqslant r$. 
Now we modify $h_{i}$ on a set of measure zero by introducing

$$
H_{i}(r)=\sup \left\{h_{i}(R): R>r \text { and } R \in(0, \infty) \backslash \Gamma\right\} .
$$

It follows that $H_{i}$ is non-negative, non-increasing and right-continuous on $(0, \infty)$ and that there is a subset $\Phi$ of one-dimensional measure zero such that $\Gamma \subset \Phi$ and $H_{i}(r)=h_{i}(r)$ for all $r \in(0, \infty) \backslash \Phi$. Setting

$$
G_{i}(x)=H_{i}(|x|)-L_{i} \quad \text { for } x \in \mathbb{R}^{N} \backslash\{0\},
$$

where $L_{i}=\lim _{r \rightarrow \infty} H_{i}(r)$, we see that $G_{i}$ is Schwarz symmetric and that $G_{i}=g_{i}-L_{i}$ a.e. on $\mathbb{R}^{N}$. Recalling (5.3) and (5.4), we have

$$
\int F(|x|, u(x)) d x=\sum_{i=0}^{k} \int\left[G_{i}(x)+L_{i}\right] \chi_{S_{i}}(x) d x
$$

and by Proposition 3.3,

$$
\int G_{i}(x) \chi_{S_{i}}(x) d x \leqslant \int G_{i}^{*}(x)\left(\chi_{S_{i}}\right)^{*}(x) d x
$$

where $G_{i}=G_{i}^{*}$ by part (iii) of Proposition 2.4 and $\left(\chi_{S_{i}}\right)^{*}=\chi_{B_{i}}$ by (3.7). Thus

$$
\int F(|x|, u(x)) d x \leqslant \int \sum_{i=0}^{k}\left[G_{i}(x)+L_{i}\right] \chi_{B_{i}}(x) d x=\int \sum_{i=0}^{k} g_{i}(x) \chi_{B_{i}}(x) d x
$$

since $\mu\left(S_{i}\right)=\mu\left(B_{i}\right)$.

On the other hand, using the notation introduced in (3.3), we have

$$
\int F\left(|x|, u^{*}(x)\right) d x=\int F\left(|x|, \sum_{i=0}^{k} a_{i} \chi_{C_{i}}(x)\right) d x=\int \sum_{i=0}^{k} F\left(|x|, a_{i}\right) \chi_{C_{i}}(x) d x
$$

since $F(|x|, 0)=0$ and $C_{i} \cap C_{j}=\emptyset$ for $i \neq j$. Now by (3.4),

$$
\sum_{i=0}^{k} F\left(|x|, a_{i}\right) \chi_{C_{i}}(x)=\sum_{i=0}^{k} g_{i}(x) \chi_{B_{i}}(x)
$$

since $B_{i}=\bigcup_{j=0}^{i} C_{j}$ where the functions $g_{i}$ are the same as in (5.4). Hence

$$
\int F\left(|x|, u^{*}(x)\right) d x=\int \sum_{i=0}^{k} g_{i}(x) \chi_{B_{i}}(x) d x .
$$

Comparing (5.5) and (5.6), we see that (1.3) holds for all $u \in E_{N}$. Using Proposition 3.2 and Proposition 2.4(v), the Monotone Convergence Theorem (see [9, Theorem 27.B], for example) yields the same inequality for all $u \in F_{N}$.

For dealing with the energy functional of a differential equation like (1.1), we formulate the following result which is an immediate consequence of Proposition 5.1.

Corollary 5.2. Let $f:(0, \infty) \times[0, \infty) \rightarrow \mathbb{R}$ be a Carathéodory function such that

(i) $f(r, s) \geqslant 0$ for all $r \in(0, \infty) \backslash \Gamma$ and all $s \geqslant 0$,

(ii) $f(r, s) \geqslant f(R, s)$ for all $r, R \in(0, \infty) \backslash \Gamma$ with $R \geqslant r$ and all $s \geqslant 0$. 
Let $F:(0, \infty) \times[0, \infty) \rightarrow \mathbb{R}$ be such that

$$
F(r, s)=\int_{0}^{s} f(r, t) d t \quad \text { for } r \in(0, \infty) \backslash \Gamma \text { and } s \geqslant 0 .
$$

Then the inequality (1.3) holds for all $u \in F_{N}$.

We now show how the hypotheses of Proposition 5.1 can be relaxed by making use of (4.1). Recall that an extended real-valued function $w: \mathbb{R}^{N} \rightarrow \mathbb{R}$ is said to be integrable if both of the functions $w_{+}$and $w_{-}$have a finite integral over $\mathbb{R}^{N}$.

THEorem 5.3. Let $F:(0, \infty) \times[0, \infty) \rightarrow \mathbb{R}$ be a Carathéodory function such that

(i) $F(|x|, 0)$ is integrable on $\mathbb{R}^{N}$,

(ii) for all $r, R \in(0, \infty) \backslash \Gamma$ with $R \geqslant r$ and all $a, b \in[0, \infty)$ with $b \geqslant a$,

$$
F(R, b)-F(R, a)-F(r, b)+F(r, a) \leqslant 0,
$$

(iii) there exists a continuous function $G:[0, \infty) \rightarrow \mathbb{R}$ such that $G(0)=0$ and

$$
\lim _{r \rightarrow \infty}\{F(r, a)-F(r, b)\} \leqslant G(a)-G(b)
$$

for all $a, b \in[0, \infty)$ with $b \geqslant a$.

Then the inequalities

$$
-\infty<\int F(|x|, u(x)) d x \leqslant \int F\left(|x|, u^{*}(x)\right) d x
$$

hold for all $u \in F_{N}$ such that $G(u)$ is integrable on $\mathbb{R}^{N}$.

Remark 1. The assumption (ii) in this result is the same as in Proposition 5.1 and it implies that, for fixed $a, b \in[0, \infty)$ with $b \geqslant a,\{F(r, a)-F(r, b)\}$ is a non-decreasing function of $r$. Thus the limit in (iii) exists and

$$
\{F(r, a)-F(r, b)\} \leqslant \lim _{r \rightarrow \infty}\{F(r, a)-F(r, b)\} \quad \text { for all } r \in(0, \infty) \backslash \Gamma .
$$

REMARK 2. If $\lim _{r \rightarrow \infty} F(r, s)=F^{\infty}(s)$ exists for all $s \geqslant 0$ and $F^{\infty}:[0, \infty) \rightarrow \mathbb{R}$ is a continuous function, then the condition (iii) is satisfied by setting $G=F^{\infty}$.

Proof. We consider the function $\Phi:(0, \infty) \times[0, \infty) \rightarrow \mathbb{R}$ defined by

$$
\Phi(r, s)=F(r, s)-F(r, 0)-G(s) .
$$

This is a Carathéodory function which satisfies the hypotheses (i) and (ii) of Proposition 5.1. But, for $r \in(0, \infty) \backslash \Gamma$ and $a, b \in[0, \infty)$ with $b \geqslant a$,

$$
\begin{aligned}
\Phi(r, a)-\Phi(r, b) & =F(r, a)-F(r, b)-\{G(a)-G(b)\} \\
& \leqslant \lim _{r \rightarrow \infty}\{F(r, a)-F(r, b)\}-\{G(a)-G(b)\} \leqslant 0
\end{aligned}
$$

by the monotonicity of $F(r, a)-F(r, b)$ and the assumption (iii). Thus we see that $\Phi$ satisfies all the conditions of Proposition 5.1 and so

$$
0 \leqslant \int \Phi(|x|, u(x)) d x \leqslant \int \Phi\left(|x|, u^{*}(x)\right) d x \text { for all } u \in F_{N},
$$


that is to say,

$$
\begin{aligned}
0 & \leqslant \int\{F(|x|, u(x))-F(|x|, 0)-G(u(x))\} d x \\
& \leqslant \int\left\{F\left(|x|, u^{*}(x)\right)-F(|x|, 0)-G\left(u^{*}(x)\right)\right\} d x
\end{aligned}
$$

for all $u \in F_{N}$. But, by Theorem 4.4,

$$
-\infty<\int G(u(x)) d x=\int G\left(u^{*}(x)\right) d x<\infty
$$

for all $u \in F_{N}$ such that $G(u)$ is integrable and, by hypothesis

$$
-\infty<\int F(|x|, 0) d x<\infty .
$$

The conclusion follows.

The remainder of this section deals with functions $u$ in the Lebesgue spaces $L^{p}\left(\mathbb{R}^{N}\right)$ for some $p \in[1, \infty)$, and with situations where $F(|x|, u(x))$ and $F\left(|x|, u^{*}(x)\right)$ are both integrable on $\mathbb{R}^{N}$.

Theorem 5.4. Let $F:(0, \infty) \times[0, \infty) \rightarrow \mathbb{R}$ be a Carathéodory function such that:

(i) the condition (ii) of Theorem 5.3 is satisfied, and

(ii) there exist a measurable function $a$ on $(0, \infty)$ and constants $b \geqslant 0$ and $p \geqslant 1$ such that

$$
|F(r, s)| \leqslant a(r)+b s^{p} \quad \text { for all } r>0 \text { and } s \geqslant 0
$$

where

$$
\int_{0}^{\infty} a(r) r^{N-1} d r<\infty
$$

Then

$$
-\infty<\int F(|x|, u(x)) d x \leqslant \int F\left(|x|, u^{*}(x)\right) d x<\infty
$$

for all $u \in L_{+}^{p}=\left\{u \in F_{N}: \int u(x)^{p} d x<\infty\right\}$.

REMARK 1. The set $L_{+}^{p}$ consists of the non-negative elements in the usual Lebesgue space $L^{p}\left(\mathbb{R}^{N}\right)$. As is well known, the condition (ii) is necessary and sufficient for ensuring that the composition operator $u \mapsto F(|\cdot|, u(\cdot))$ maps $L^{p}\left(\mathbb{R}^{N}\right)$ into $L^{1}\left(\mathbb{R}^{N}\right)$.

REMARK 2. To obtain the conclusion for a given element $u \in L_{+}^{p}$ rather than for all such functions, the condition (ii) can be relaxed and this will be used when we deal with functions $u$ defined on subsets of $\mathbb{R}^{N}$ in $\S 6$. Suppose that $\Omega$ is a measurable subset of $[0, \infty)$ such that

$$
u(x)=u^{*}(x)=0 \quad \text { for all } x \in \mathbb{R}^{N} \text { such that }|x| \notin \Omega .
$$


Then the hypothesis (ii) can be replaced by

(ii) $)_{u} F(\cdot, 0) \equiv 0$ and there exist a measurable function $A$ on $\Omega$ and constants $b \geqslant 0$ and $p \geqslant 1$ such that

$$
|F(r, s)| \leqslant A(r)+b s^{p} \quad \text { for all } r \in \Omega \text { and } s \geqslant 0
$$

where

$$
\int_{\Omega} A(r) r^{N-1} d r<\infty
$$

Proof. The assumption (ii) ensures that the function $F(|\cdot|, 0)$ is integrable on $\mathbb{R}^{N}$. This means that it is enough to establish the result for the function $F-F(|\cdot|, 0)$ rather than for $F$ itself. Consequently, we assume henceforth that $F(\cdot, 0) \equiv 0$.

Let

$$
K=\left\{u \in E_{N}: u \text { has compact support }\right\} .
$$

We first prove the result for elements of $K$ and then we extend the conclusion to all of $L_{+}^{p}$.

Let $u \in K$ be fixed. There are constants $T \notin \Gamma$ and $S$ (depending on $u$ ) such that

$$
u(x)=u^{*}(x)=0 \quad \text { for }|x| \geqslant T
$$

and

$$
0 \leqslant \max \left\{u(x), u^{*}(x)\right\} \leqslant S \text { for all }|x| \leqslant T .
$$

We define a function $\Phi:(0, \infty) \times[0, \infty) \rightarrow \mathbb{R}$ as follows:

$$
\Phi(r, s)= \begin{cases}F(r, s) & \text { if } r \in(0, T] \text { and } s \leqslant S, \\ F(T, s) & \text { if } r \in(T, \infty) \text { and } s \leqslant S, \\ F(r, S) & \text { if } r \in(0, T] \text { and } s>S, \\ F(T, S) & \text { if } r \in(T, \infty) \text { and } s>S .\end{cases}
$$

Clearly $\Phi:(0, \infty) \times[0, \infty) \rightarrow \mathbb{R}$ is a Carathéodory function with $\Phi(r, 0) \equiv 0$ and $\Phi(r, \cdot):[0, \infty) \rightarrow \mathbb{R}$ is continuous for all $r \in(0, \infty) \backslash \Gamma$. Furthermore, $\Phi$ satisfies the condition (ii) of Theorem 5.3.

Now set

$$
G(s)= \begin{cases}F(T, s) & \text { for } s \leqslant S, \\ F(T, S) & \text { for } s>S .\end{cases}
$$

Clearly $G:[0, \infty) \rightarrow \mathbb{R}$ is a continuous function and $G(0)=0$. Also, for all $a, b \in[0, \infty)$,

$$
\lim _{r \rightarrow \infty}\{\Phi(r, a)-\Phi(r, b)\}=G(a)-G(b),
$$

so the function $\Phi$ satisfies the condition (iii) of Theorem 5.3. Furthermore, since $u \in K$, we see that $G(u)$ is integrable on $\mathbb{R}^{N}$. Thus $\Phi$ satisfies the hypotheses of Theorem 5.3 and we obtain

$$
-\infty<\int \Phi(|x|, u(x)) d x \leqslant \int \Phi\left(|x|, u^{*}(x)\right) d x .
$$

But $\max \left\{u(x), u^{*}(x)\right\} \leqslant S$ and $(\operatorname{supp} u) \cup\left(\operatorname{supp} u^{*}\right) \subset B(0, T)$, and so this inequality 
can be written as

$$
\int F(|x|, u(x)) d x \leqslant \int F\left(|x|, u^{*}(x)\right) d x .
$$

This establishes (1.3) for all elements of $K$.

Now consider an arbitrary element $u \in L_{+}^{p}$. First we observe that

$$
\int|F(|x|, u(x))| d x \leqslant \int\left\{a(|x|)+b|u(x)|^{p}\right\} d x<\infty .
$$

Furthermore, by Proposition 3.2, there is a sequence $\left\{u_{n}\right\} \subset E_{N}$ such that $0 \leqslant u_{n} \leqslant u_{n+1}$ and $u_{n} \rightarrow u$ on $\mathbb{R}^{N}$. Let $v_{n}=u_{n} \chi_{B(0, n)}$. Now, for all $n \in \mathbb{N}$, $v_{n} \in K$ and we have

$$
0 \leqslant v_{n} \leqslant v_{n+1} \leqslant u \quad \text { and } \quad v_{n} \rightarrow u \text { on } \mathbb{R}^{N} .
$$

In particular,

$$
0 \leqslant\left|u-v_{n}\right|^{p} \leqslant 2^{p} u^{p}
$$

and so by the Dominated Convergence Theorem, $\left\|v_{n}-u\right\|_{p} \rightarrow 0$ where $\|\cdot\|_{p}$ denotes the usual norm on the Banach space $L^{p}\left(\mathbb{R}^{N}\right)$. By the basic result about Nemytskii operators (see [6, Theorem 2.3], for example), the assumption (ii) implies that

$$
\int|F(|x|, u(x))| d x<\infty \quad \text { and } \quad \int\left|F\left(|x|, v_{n}(x)\right)-F(|x|, u(x))\right| d x \rightarrow 0 .
$$

(Note that, in the context of (ii) $)_{u}, v_{n}(x)=0$ for all $x \in \mathbb{R}^{N}$ such that $|x| \notin \Omega$.) Hence,

$$
-\infty<\int F(|x|, u(x)) d x=\lim _{n \rightarrow \infty} \int F\left(|x|, v_{n}(x)\right) d x<\infty .
$$

By Proposition 2.4(v),

$$
0 \leqslant v_{n}^{*} \leqslant v_{n+1}^{*} \leqslant u^{*} \quad \text { and } \quad v_{n}^{*} \rightarrow u^{*} \quad \text { on } \mathbb{R}^{N},
$$

and, by Proposition 4.3,

$$
\int\left(u^{*}(x)\right)^{p} d x=\int(u(x))^{p} d x<\infty .
$$

As before, the Dominated Convergence Theorem shows that $\left\|v_{n}^{*}-u^{*}\right\|_{p} \rightarrow 0$ and so the continuity of the Nemytskii operator yields

$$
-\infty<\int F\left(|x|, u^{*}(x)\right) d x=\lim _{n \rightarrow \infty} \int F\left(|x|, v_{n}^{*}(x)\right) d x<\infty .
$$

But we have established that

$$
\int F\left(|x|, v_{n}(x)\right) d x \leqslant \int F\left(|x|, v_{n}^{*}(x)\right) d x \text { for all } n
$$

since $v_{n} \in K$. Combining (5.7)-(5.9) completes the proof.

We can now state a variant of Corollary 5.2 which is well adapted to dealing with energy functionals on Sobolev spaces. 
Corollary 5.5. Let $f:(0, \infty) \times[0, \infty) \rightarrow \mathbb{R}$ be a Carathéodory function such that

(i) there exist a measurable function $a$ on $(0, \infty)$ and constants $b \geqslant 0$ and $p \geqslant 1$ such that

$$
|f(r, s)| \leqslant a(r)+b s^{p-1} \quad \text { for all } r>0 \text { and } s \geqslant 0
$$

where $\int_{0}^{\infty} a(r)^{q} r^{N-1} d r<\infty$ with $1 / q+1 / p=1$ if $p>1$ and $a(r) \equiv 0$ if $p=1$ (in this case $f$ is bounded),

(ii) $f(r, s) \geqslant f(R, s)$ for all $r, R \in(0, \infty) \backslash \Gamma$ with $R \geqslant r$ and all $s \geqslant 0$.

Let $F:(0, \infty) \times[0, \infty) \rightarrow \mathbb{R}$ be such that

$$
F(r, s)=\int_{0}^{s} f(r, t) d t \quad \text { for } r \in(0, \infty) \backslash \Gamma \text { and } s \geqslant 0 .
$$

Then

$$
-\infty<\int F(|x|, u(x)) d x \leqslant \int F\left(|x|, u^{*}(x)\right) d x<\infty
$$

holds for all $u \in L_{+}^{p}$.

For smooth functions the condition (ii) in Proposition 5.1 and Theorem 5.3 is essentially equivalent to the condition $\partial_{1} \partial_{2} F \leqslant 0$. This observation yields the following result.

THEOREM 5.6. Suppose that $F$ has the following properties:

(i) $F \in C^{2}([0, \infty) \times[0, \infty))$ with $\partial_{1} \partial_{2} F(r, s) \leqslant 0$ for all $r, s \geqslant 0$;

(ii) there exist a measurable function $a$ on $(0, \infty)$ and constants $b \geqslant 0$ and $p \geqslant 1$ such that

$$
|F(r, s)| \leqslant a(r)+b s^{p} \quad \text { for all } r>0 \text { and } s \geqslant 0
$$

where $\int_{0}^{\infty} a(r) r^{N-1} d r<\infty$.

Then

$$
-\infty<\int F(|x|, u(x)) d x \leqslant \int F\left(|x|, u^{*}(x)\right) d x<\infty
$$

for all $u \in L_{+}^{p}$.

Proof. The hypotheses of Theorem 5.4 are clearly satisfied.

\section{Symmetrization on finite subsets of $\mathbb{R}^{N}$}

So far we have considered only the symmetrization of functions defined on all of $\mathbb{R}^{N}$. However it is easy enough to derive results about functions defined on bounded sets from this case.

More generally, let $\omega$ be a measurable subset of $\mathbb{R}^{N}$ which has finite measure and let $\omega^{*}=B(0, T)$. Let $F_{N}(\omega)$ denote the set of all extended real-valued functions $u$ such that

(i) $u$ is measurable on $\omega$ and

(ii) $0 \leqslant u<\infty$ a.e. on $\omega$. 
Clearly, $\left.f\right|_{\omega} \in F_{N}(\omega)$ for all $f \in F_{N}$ and, conversely, given any $f \in F_{N}(\omega)$ we have $\widetilde{f} \in F_{N}$ where $\widetilde{f}$ is defined by

$$
\widetilde{f}= \begin{cases}f(x) & \text { if } x \in \omega, \\ 0 & \text { if } x \in \mathbb{R}^{N} \backslash \omega .\end{cases}
$$

The Schwarz symmetrization of an element of $F_{N}(\omega)$ can be defined as follows.

Definition 6.1. Given $u \in F_{N}(\omega)$, the Schwarz symmetrization of $u$ is the function $u^{*}$ defined on $\omega^{*}$ by

$$
u^{*}=\left.\left[(\widetilde{u})^{*}\right]\right|_{\omega^{*}}
$$

where $\widetilde{u}$ is the extension of $u$ to all of $\mathbb{R}^{N}$ defined by (6.1).

Let us note some important properties of $u^{*}$.

Lemma 6.1. For any $u \in F_{N}(\omega)$,

(i) $(\widetilde{u})^{*}(x)=0$ for all $x \in \mathbb{R}^{N} \backslash \omega^{*}$,

(ii) for all $t \geqslant 0, \mu(\{x \in \omega: u(x)>t\})=\mu\left(\left\{x \in \omega^{*}: u^{*}(x)>t\right\}\right)$, and

(iii) $\mu(\{x \in \omega: u(x)=0\})=\mu\left(\left\{x \in \omega^{*}: u^{*}(x)=0\right\}\right)$.

Proof. (i) For all $t \geqslant 0,\left\{x \in \mathbb{R}^{N}: \widetilde{u}(x)>t\right\}=\{x \in \omega: u(x)>t\}$ and so

$$
d_{\widetilde{u}}(t)=\mu\left(\left\{x \in \mathbb{R}^{N}: \widetilde{u}(x)>t\right\}\right) \leqslant \mu(\omega)=V_{N} T^{N} .
$$

Hence, $h_{\widetilde{u}}(s)=0$ for all $s \geqslant T$. Thus $(\widetilde{u})^{*}(x)=0$ for all $x \notin B(0, T)=\omega^{*}$.

(ii) For all $t \geqslant 0$,

and, by part (i),

$$
\{x \in \omega: u(x)>t\}=\left\{x \in \mathbb{R}^{N}: \widetilde{u}(x)>t\right\}
$$

$$
\left\{x \in \mathbb{R}^{N}:(\widetilde{u})^{*}(x)>t\right\}=\left\{x \in \omega^{*}:(\widetilde{u})^{*}(x)>t\right\}=\left\{x \in \omega^{*}: u^{*}(x)>t\right\} .
$$

But, by Proposition 2.4,

$$
\mu\left(\left\{x \in \mathbb{R}^{N}: \widetilde{u}(x)>t\right\}\right)=\mu\left(\left\{x \in \mathbb{R}^{N}:(\widetilde{u})^{*}(x)>t\right\}\right),
$$

yielding the desired result.

(iii) We have

$$
\begin{aligned}
\mu(\{x \in \omega: u(x)=0\}) & =\mu(\omega \backslash\{x \in \omega: u(x)>0\}) \\
& =\mu(\omega)-\mu(\{x \in \omega: u(x)>0\})
\end{aligned}
$$

and similarly,

$$
\mu\left(\left\{x \in \omega^{*}: u^{*}(x)=0\right\}\right)=\mu\left(\omega^{*}\right)-\mu\left(\left\{x \in \omega^{*}: u^{*}(x)>0\right\}\right) .
$$

The result follows using part (ii).

Remark. As we showed using the function (4.3), the equality in part (iii) can fail when $\omega$ has infinite measure.

For functions $u \in F_{N}(\omega)$ we can now obtain results analogous to those already established for functions $u \in F_{N}$. We give some typical examples. The first one 
deals with composition with a Borel function. In keeping with Lemma 6.1(iii), it turns out that the restriction $G(0)=0$ which we required in $\S 4$ is no longer necessary. Here we recover the result in the form established in [13]. See also [11] for the case where $G$ is continuous.

Theorem 6.2. Let $G:[0, \infty) \rightarrow \mathbb{R}$ be a Borel measurable function. Then the equality

$$
\int_{\omega} G(u(x)) d x=\int_{\omega^{*}} G\left(u^{*}(x)\right) d x
$$

holds for all $u \in F_{N}(\omega)$ such that at least one of the integrals $\int_{\omega} G_{+}(u(x)) d x$ and $\int_{\omega} G_{-}(u(x)) d x$ is finite.

Proof. Let $g(s)=G(s)-G(0)$. It follows from Theorem 4.4 that

$$
\int g(\widetilde{u}(x)) d x=\int g\left((\widetilde{u})^{*}(x)\right) d x
$$

provided that at least one of the integrals $\int g_{+}(\widetilde{u}(x)) d x$ and $\int g_{-}(\widetilde{u}(x)) d x$ is finite. But

$$
g_{+}(s)=\max \{G(s)-G(0), 0\} \leqslant \max \left\{G_{+}(s)+G_{-}(0), 0\right\}=G_{+}(s)+G_{-}(0) .
$$

Therefore, since $g(0)=0$,

$$
\int g_{+}(\widetilde{u}(x)) d x=\int_{\omega} g_{+}(u(x)) d x \leqslant \int_{\omega} G_{+}(u(x)) d x+G_{-}(0) \mu(\omega) .
$$

Thus, if $\int_{\omega} G_{+}(u(x)) d x$ is finite, then $\int g_{+}(\widetilde{u}(x)) d x$ is also finite and we can conclude that (6.3) holds. That is to say, using Lemma 6.1(i), we have

$$
\int_{\omega} g(\widetilde{u}(x)) d x=\int g(\widetilde{u}(x)) d x=\int g\left((\widetilde{u})^{*}(x)\right) d x=\int_{\omega^{*}} g\left((\widetilde{u})^{*}(x)\right) d x
$$

since $g(0)=0$. Hence

$$
\begin{aligned}
\int_{\omega}\{G(\widetilde{u}(x))-G(0)\} d x & =\int_{\omega} g(\widetilde{u}(x)) d x \\
& =\int_{\omega^{*}} g\left((\widetilde{u})^{*}(x)\right) d x=\int_{\omega^{*}}\left\{G\left((\widetilde{u})^{*}(x)\right)-G(0)\right\} d x,
\end{aligned}
$$

which yields (6.2) in the case when $\int_{\omega} G_{+}(u(x)) d x$ is finite.

If $\int_{\omega} G_{+}(u(x)) d x=\infty$, then $\int_{\omega} G_{-}(u(x)) d x$ is finite. Noting that

$g_{-}(s)=\max \{-G(s)+G(0), 0\} \leqslant \max \left\{G_{-}(s)+G_{+}(0), 0\right\}=G_{-}(s)+G_{+}(0)$, we find that

$$
\int g_{-}(\widetilde{u}(x)) d x=\int_{\omega} g_{-}(u(x)) d x \leqslant \int_{\omega} G_{-}(u(x)) d x+G_{+}(0) \mu(\omega)<\infty,
$$

and the proof can be completed as in the previous case.

THEOREM 6.3. Let $F$ satisfy the conditions (ii) and (iii) of Theorem 5.3 and suppose that $F(|x|, 0)$ is integrable on $\omega$ and on $\omega^{*}$ with

$$
\int_{\omega} F(|x|, 0) d x \leqslant \int_{\omega^{*}} F(|x|, 0) d x .
$$


The inequalities

$$
-\infty<\int_{\omega} F(|x|, u(x)) d x \leqslant \int_{\omega^{*}} F\left(|x|, u^{*}(x)\right) d x
$$

hold for all $u \in F_{N}(\omega)$ such that $G(u)$ is integrable on $\omega$.

REMARK. The condition (6.4) is clearly necessary. Furthermore, the restriction that $G(0)=0$ in condition (iii) of Theorem 5.3 now involves no loss of generality since we can replace $G$ by $G-G(0)$ without influencing the integrability of $G(u)$.

Proof. Setting $\Phi(r, s)=F(r, s)-F(r, 0)$, we see that $\Phi$ satisfies all the hypotheses of Theorem 5.3 with the same function $G$. Thus

$$
\int G_{ \pm}(\widetilde{u}(x)) d x=\int_{\omega} G_{ \pm}(u(x)) d x
$$

showing that $G(\widetilde{u})$ is integrable on $\mathbb{R}^{N}$. Hence, by Theorem 5.3,

$$
-\infty<\int \Phi(|x|, \widetilde{u}(x)) d x \leqslant \int \Phi\left(|x|,(\widetilde{u})^{*}(x)\right) d x
$$

which can be written as

$$
-\infty<\int_{\omega}\{F(|x|, u(x))-F(|x|, 0)\} d x \leqslant \int_{\omega^{*}}\left\{F\left(|x|, u^{*}(x)\right)-F(|x|, 0)\right\} d x .
$$

This yields the desired inequality since $F(|x|, 0)$ is integrable on $\omega$ and $\omega^{*}$ and (6.4) holds.

Finally we note that we can easily recover the result in [16] concerning smooth functions $F$ which we mentioned in $\S 1$. The hypothesis

$$
F(r, s) \text { is non-increasing with respect to } r \text {, }
$$

which was added in the Corrigendum to [16], implies that our condition (6.4) is satisfied.

THEOREM 6.4. Let $F:(0, \infty) \times[0, \infty) \rightarrow \mathbb{R}$ be a Carathéodory function satisfying (6.4) and the condition (ii) of Theorem 5.3. Let $\Omega=\left\{|x|: x \in \omega \cup \omega^{*}\right\}$ and suppose that there exist a measurable function $A$ on $\Omega$ and constants $b \geqslant 0$ and $p \geqslant 1$ such that

$$
|F(r, s)| \leqslant A(r)+b s^{p} \quad \text { for all } r \in \Omega \text { and } s \geqslant 0
$$

where

$$
\int_{\Omega} A(r) r^{N-1} d r<\infty
$$

Then

$$
-\infty<\int_{\omega} F(|x|, u(x)) d x \leqslant \int_{\omega^{*}} F\left(|x|, u^{*}(x)\right) d x<\infty
$$

for all $u \in L_{+}^{p}(\omega)=\left\{u \in L^{p}(\omega): u \geqslant 0\right.$ a.e. on $\left.\omega\right\}$.

Proof. Let $\Phi(r, s)=F(r, s)-F(r, 0)$. For any $u \in L_{+}^{p}(\omega)$, it is clear that $\widetilde{u} \in L_{+}^{p}$ and that

$$
\widetilde{u}(x)=(\widetilde{u})^{*}(x)=0 \quad \text { for all } x \in \mathbb{R}^{N} \text { such that }|x| \notin \Omega
$$


Hence $\Phi$ satisfies the condition (ii) $\widetilde{u}$ that was introduced in the Remark 2 following Theorem 5.4. But $\Phi:(0, \infty) \times[0, \infty) \rightarrow \mathbb{R}$ is clearly a Carathéodory function satisfying the condition (i) of Theorem 5.4. Hence,

$$
-\infty<\int \Phi(|x|, \widetilde{u}(x)) d x \leqslant \int \Phi\left(|x|,(\widetilde{u})^{*}(x)\right) d x<\infty .
$$

But, since $\Phi(r, 0) \equiv 0$, we find that

$$
\int \Phi(|x|, \widetilde{u}(x)) d x=\int_{\omega}\{F(|x|, u(x))-F(|x|, 0)\} d x
$$

and, using Lemma 6.1, we have

$$
\int \Phi\left(|x|,(\widetilde{u})^{*}(x)\right) d x=\int_{\omega^{*}}\left\{F\left(|x|, u^{*}(x)\right)-F(|x|, 0)\right\} d x .
$$

Now $|F(r, 0)| \leqslant A(r)$ and so $F(|x|, 0)$ is integrable on $\omega$ and on $\omega^{*}$. Using (6.4), we have the required result.

\section{References}

1. C. BANDLE, Isoperimetric inequalities and applications (Pitman, London, 1980).

2. F. BRock, 'Continuous rearrangement and symmetry of solutions of elliptic problems', Proc. Indian Acad. Sci. Math. Sci. 110 (2000) 157-204.

3. F. BRock, 'A general rearrangement inequality à la Hardy-Littlewood', J. Inequal. Appl. 5 (2000) 309-320.

4. F. Brock and A. Solynin, 'An approach to symmetrization via polarization', Trans. Amer. Math. Soc. 352 (1996) 1759-1796.

5. J. A. Crowe, P. C. Rosenbloom and J. A. Zweibel, 'Rearrangement of functions', J. Funct. Anal. 66 (1986) 432-438.

6. D. G. DE Figueiredo, The Ekelend variational principle with applications and detours, Tata Institute Notes (Springer, Berlin, 1989).

7. G. B. Folland, Real analysis, 2nd edn (Wiley, New York, 1999).

8. H. Hajaiej, 'Inégalités de symétrisation et applications', Thèse 2465, EPFL, Lausanne, 2001.

9. P. R. Halmos, Measure theory, Graduate Texts in Mathematics (Springer, Berlin, 1950).

10. O. Kavian, Introduction à la théorie des points critiques, Série Mathématiques et Applications 13 (Springer, Berlin, 1993).

11. B. KAWOHL, On rearrangements, symmetrization and maximum principles, Lecture Notes in Mathematics 1150 (Springer, Berlin, 1985).

12. E. H. Lieb and M. Loss, Analysis, Graduate Studies in Mathematics 14 (American Mathematical Society, Providence, RI, 1997).

13. J. Mossino, Inégalités isopérimétriques et applications en physique (Hermann, Paris, 1984).

14. C. A. Stuart, 'A variational approach to bifurcation in $L^{p}$ on an unbounded symmetrical domain', Math. Ann. 263 (1983) 51-59.

15. C. A. Stuart, 'Bifurcation in $L^{p}\left(\mathbb{R}^{N}\right)$ for a semilinear elliptic equation', Proc. London Math. Soc. (3) 57 (1988) 511-541.

16. R. TahraouI, 'Symmetrization inequalities', Nonlinear Anal. 27 (1996) 933-955, Corrigendum, ibid. 33 (2000) 535.

H. Hajaiej and C. A. Stuart

IACS-FSB

Section de Mathématiques

École Polytechnique Fédérale de Lausanne

CH-1015 Lausanne

Switzerland

hichem.hajaiej@epfl.ch

charles.stuart@epfl.ch 